\title{
Differences between Istradefylline Responders and Non-Responders in Parkinson's Disease
}

\author{
Asako Yoritaka1, Nobutaka Hattori² \\ ${ }^{1}$ Department of Neurology, Juntendo University Koshigaya Hospital, Saitama, Japan \\ ${ }^{2}$ Department of Neurology, Juntendo University School of Medicine, Tokyo, Japan \\ Email: ayori@juntendo.ac.jp, nhattori@juntendo.ac.jp
}

How to cite this paper: Yoritaka, A. and Hattori, N. (2017) Differences between Istradefylline Responders and Non-Responders in Parkinson's Disease. Advances in Parkinson's Disease, 6, 45-51.

https://doi.org/10.4236/apd.2017.62005

Received: February 10, 2017

Accepted: March 28, 2017

Published: March 31, 2017

Copyright ( 92017 by authors and Scientific Research Publishing Inc. This work is licensed under the Creative Commons Attribution International License (CC BY 4.0).

http://creativecommons.org/licenses/by/4.0/

\section{c) (i) Open Access}

\begin{abstract}
Background: Istradefylline is a selective adenosine A2A receptor antagonist approved for Parkinson's disease (PD) patients with wearing-off symptoms. The Japanese phase III trial showed that $20 \mathrm{mg}$ of orally administrated istradefylline decreased the Off-time. However, istradefylline showed prominent effects in some patients and no benefits in others. We examined the differences in characteristics between responders and non-responders who received 8 weeks of $20 \mathrm{mg} /$ day istradefylline. Methods: Thirty-one patients were enrolled (age, 65.4 [SD 10.4] years; disease duration, 10.4 [SD 6.1] years; daily levodopa dosage, 553.2 [SD 228.7] mg; frequency of levodopa consumption, 4.7 [SD 1.5] times; levodopa equivalent dose, 811.2 [SD 307.5] mg). Results: There were significant differences $(\mathrm{p}<0.05)$ in sex (male/female: $5 / 16,6 / 4$ ), age (62.9 (SD 10.4), 70.6 (SD 8.0) years), age at onset (51.9 (SD 12.3), 61.5 (SD 10.5) years old), age at dyskinesia onset (57.9 (SD 8.8), 67.6 (SD 7.2), and Epworth sleepiness scale scores (4.5 (SD 2.7), 11.2 (SD 6.7), $\mathrm{p}<0.01$ ) for the responders and non-responders, respectively. There were no differences in disease duration, On-time, Off-time, Unified Parkinson's disease Rating scale scores, daily levodopa dose, levodopa equivalent dose, cumulative levodopa dose, or coffee intake. Conclusions: Younger or female patients who are not excessively sleepy during daytime are better candidates for the istradefylline therapy.
\end{abstract}

\section{Keywords}

Parkinson's Disease, Istradefylline, Adenosine A2A Receptor Antagonist, Wearing-Off, Sleepiness

\section{Introduction}

Current treatments for Parkinson's disease (PD) are based on dopamine replacement therapy, including L-3, 4-dihydroxyphenylalnine (levodopa, L-DOPA), 
dopamine agonists (DA), monoamine oxidase B inhibitors (MAOB-I), and cathechol-O-methyltransferase inhibitors (COMT-I). Istradefylline is a selective antagonist of the adenosine $\mathrm{A} 2 \mathrm{~A}$ receptor, and has been approved for use in $\mathrm{PD}$ with regard to anti-parkinsonian effects in patients with wearing-off symptoms [1]. A recent phase III trial demonstrated that the oral administration of istradefylline $(20 \mathrm{mg}$ ) reduced off time in patients, while $40 \mathrm{mg}$ of istradefylline improved part III of the Unified Parkinson's disease Rating scale (UPDRS) during on-phase [2]. However, the clinical results were inconsistent. While istradefylline demonstrated prominent effects in a number of patients; others experienced little or no benefit. Therefore, to elucidate the epidemiological differences between istradefylline responders and non-responders, a retrospective analysis was performed.

\section{Methods and Materials}

\subsection{Patients}

Patients with PD were recruited according to the following specifications: 1) patients were diagnosed on the basis of the UK PD Brain Bank criteria [3]; 2) patients were medicated with levodopa and experienced wearing off; 3) patients featured a rating between 0 and 4 on the modified Hoehn and Yahr (H\&Y) scale during the on-phase; and 4) patients wanted to reduce their off-phase disability and received $20 \mathrm{mg} /$ day of istradefylline once in the morning in A.Y.'s outpatient clinic at the Juntendo University Koshigaya hospital between October 2013 and October 2015. Participants were excluded based on the following criteria: 1) patients experienced Parkinsonism due to diseases other than PD;2) patients were diagnosed with other serious diseases, malignant tumors, or adverse events caused by drugs. All patients provided informed consent prior to the data collection. This study was approved by the Juntendo Koshigaya Hospital Institutional Ethics Committee.

\subsection{Evaluations}

Upon initiation of istradefylline (baseline) treatment and after the $8^{\text {th }}$ week visit, patients were examined according to the UPDRS [4], Epworth sleepiness scale (ESS), [5] on time and off time, and sleeping time, which were recorded in an on/off hourly diary for one week. Responders were evaluated according to the criteria: "very much improved," "much improved," and "minimally improved," while non-responders were defined as "no change" and "worsened" using the Patients Global Impression of Improvement.

\subsection{Statistical Analysis}

We compared the two groups by using either t-test or $\chi^{2}$-test using SPSS (Version 20.0). Two-sided statistical tests were used and the significance level was set at 0.05 .

\section{Results}

The epidemiological characteristics of 31 Japanese patients (male 11, female 20) 
Table 1. Patient characteristics at baseline and the $8^{\text {th }}$ week.

\begin{tabular}{|c|c|c|c|c|c|c|}
\hline & & \multicolumn{2}{|c|}{ Baseline } & \multicolumn{2}{|c|}{ 8th week } & \multirow[b]{2}{*}{$p$} \\
\hline & & Mean & $\begin{array}{c}\text { Std. } \\
\text { Deviation }\end{array}$ & Mean & $\begin{array}{c}\text { Std. } \\
\text { Deviation }\end{array}$ & \\
\hline Age & & 65.4 & 10.3 & & & \\
\hline Disease duration (years) & & 10.4 & 6.1 & & & \\
\hline Daily levodopa (mg) & & 553.2 & 228.7 & & & \\
\hline Frequency of the levodopa consumptio & & 4.7 & 1.5 & & & \\
\hline Levodopa equivalent dose (mg) & & 811.2 & 307.5 & & & \\
\hline \multicolumn{7}{|l|}{ Unified Parkinson's disease Rating scale } \\
\hline Total & 32.1 & & 24.2 & 28.5 & 21.4 & $0.030^{*}$ \\
\hline Part II & 8.3 & & 7.2 & 6.9 & 5.7 & $0.015^{*}$ \\
\hline Part III & 19.4 & & 16.9 & 17.2 & 15.3 & 0.107 \\
\hline Part IV & 3.9 & & 1.9 & 3.7 & 2.6 & 0.897 \\
\hline On time (hours) & 12.0 & & 3.4 & 14.0 & 3.4 & $0.008^{* *}$ \\
\hline Off time (hours) & 5.3 & & 3.1 & 3.1 & 2.8 & $0.007^{* *}$ \\
\hline Epworth sleepiness scale & 7.0 & & 5.6 & 6.1 & 5.3 & 0.458 \\
\hline
\end{tabular}

are displayed in Table 1 . Istradefylline significantly increased On-time $(\mathrm{p}<$ 0.01 ), and decreased Off-time ( $\mathrm{p}<0.01$ ), total UPDRS ( $\mathrm{p}<0.05$ ), and UPDRS part II $(\mathrm{p}<0.05)$ in patients with PD associated motor complications. Twenty one patients were responsive to istradefylline, while 10 patients did not demonstrate any therapeutic benefit. One patient who experienced auditory hallucinations was included in the non-responder group. The dyskinesia severity score (UPDRS) increased from 1 to 2 in several of the responders $(n=2)$. Other responders experienced slight euphoria $(n=2)$. Subsequent analysis identified significant differences in the baseline characteristics of responders $(n=21)$ and non-responders $(\mathrm{n}=10)$ with regard to sex, age, age at onset, age at dyskinesia onset, and ESS (Table 2). No differences were detected in disease duration or daily levodopa dosage. The ESS of non-responders was improved by istradefylline treatment, but was not significant. Regression analysis of changes in On- or Off-time did not indicate any correlations, and logistic regression analysis demonstrated that the odds ratio for non-responders was 1.519 (95\% confidence interval $1.021-2.261, \mathrm{p}=0.039)$ in ESS. No correlation was detected between the effects of istradefylline and the therapeutic combination of DA, COMT-I, selegiline, and zonisamide. In the non-responder group, with the exception of one patient with hallucinations, no improvements were detected after istradefylline treatment with $40 \mathrm{mg}$.

\section{Discussion}

This study demonstrated the therapeutic efficacy of istradefylline with regard to increasing On-time and reducing Off-time, and UPDRS-ADL (part II) score 
Table 2. Clinical differences of responders and non-responders to istradefylline.

\begin{tabular}{|c|c|c|c|c|c|}
\hline \multirow[b]{2}{*}{ Sex (Male: Female) n } & \multicolumn{2}{|c|}{ Responders } & \multicolumn{2}{|c|}{ Non-responders } & \multirow{2}{*}{$\frac{p}{0.049^{*}}$} \\
\hline & 5 & 16 & 6 & 4 & \\
\hline & Mean & $\begin{array}{c}\text { Std. } \\
\text { Deviation }\end{array}$ & Mean & $\begin{array}{c}\text { Std. } \\
\text { Deviation }\end{array}$ & $p$ \\
\hline Age at onset & 51.9 & 12.3 & 61.5 & 10.5 & $0.043^{*}$ \\
\hline Age at examination & 62.9 & 10.4 & 70.6 & 8.0 & $0.049^{*}$ \\
\hline Disease duration (year) & 9.1 & 6.2 & 9.1 & 6.2 & 0.429 \\
\hline Age at onset of wearing off & 59.3 & 11.0 & 65.6 & 9.4 & 0.127 \\
\hline Wearing off duration (year) & 3.7 & 3.7 & 4.1 & 4.7 & 0.774 \\
\hline Age at onset of dyskinesia & 57.9 & 8.8 & 67.6 & 7.2 & $0.025^{*}$ \\
\hline Dyskinesia duration (year) & 4.2 & 4.3 & 3.7 & 5.6 & 0.468 \\
\hline Daily cups of coffee & 1.1 & 1.2 & 0.5 & 0.6 & 0.105 \\
\hline Daily levodopa (mg) & 538.1 & 254.9 & 585.0 & 168.4 & 0.414 \\
\hline Levodopa cumulative dose (g) & 1163.5 & 1141.4 & 1023.7 & 1453.9 & 0.772 \\
\hline Levodopa equivalent dose (mg) [6] & 802.5 & 320.1 & 829.4 & 294.7 & 0.825 \\
\hline Frequency of the levodopa & 4.6 & 1.4 & 4.9 & 1.7 & 0.569 \\
\hline On time (hour) & 11.8 & 3.6 & 10.0 & 3.7 & 0.236 \\
\hline Off time (hour) & 5.3 & 3.6 & 7.1 & 2.1 & 0.216 \\
\hline Sleeping time (hour) & 6.8 & 1.9 & 6.9 & 2.3 & 0.886 \\
\hline Total UPDRS & 29.2 & 22.4 & 35.4 & 26.1 & 0.744 \\
\hline UPDRS part II & 7.9 & 7.3 & 9.6 & 7.1 & 0.617 \\
\hline UPDRS part III & 16.9 & 15.6 & 21.3 & 18.4 & 0.763 \\
\hline UPDRS part IV & 3.5 & 1.8 & 4.0 & 2.0 & 0.337 \\
\hline Epworth sleepiness scale & 4.5 & 2.7 & 11.2 & 6.7 & $0.001^{\star *}$ \\
\hline \multicolumn{6}{|l|}{ The change from the baseline } \\
\hline On time (hour) & 2.8 & 3.5 & 0.0 & 0.6 & 0.073 \\
\hline Off time (hour) & -2.8 & 3.6 & -0.3 & 0.9 & 0.105 \\
\hline Total UPDRS & -3.9 & 7.0 & -2.9 & 6.1 & 0.744 \\
\hline UPDRS part II & -1.2 & 2.4 & -1.7 & 1.9 & 0.617 \\
\hline UPDRS part III & -2.6 & 6.5 & -1.7 & 5.0 & 0.763 \\
\hline UPDRS part IV & -0.3 & 0.9 & 0.6 & 2.0 & 0.337 \\
\hline Epworth sleepiness scale & 0.0 & 1.8 & -1.8 & 4.7 & 0.231 \\
\hline
\end{tabular}

UPDRS: Unified Parkinson's disease Rating scale, ${ }^{\star} \mathrm{p}<0.05,{ }^{* *} \mathrm{p}<0.01$.

without affecting sleepiness. The majority of anti-parkinsonian drugs produce sleepiness in patients, excluding anti-cholinergics, amantagine, and selegiline; however, istradefylline maintains the level of sleepiness. Significant differences were detected between responders and non-responders in relation to sex, age, age at onset, and ESS. The study found no differences between the groups with 
regard to disease duration, daily or cumulative levodopa dose, duration of baseline On- or Off-time, or the age at the onset of wearing off. In addition, no differences were detected in the duration of dyskinesia between the two groups; however, the onset of dyskinesia was earlier than that of wearing off in responders. This might relate to the increase in putamen adenosine $A_{2 A}$ receptors observed in PD patients with dyskinesia [7].

Caffeine is an adenosine $A_{2 A}$ antagonist that induces wakefulness via pre adenosine $A_{2 \mathrm{~A}}$ receptors [8]. In the present study, the level of sleepiness correlated with the effects of istradefylline; therefore, patients who maintain sleep control might be suitable for istradefylline therapy.

Patients with $\mathrm{PD}$ demonstrate adenosine $\mathrm{A}_{2 \mathrm{~A}}$ receptor up-regulation in lymphocyte membranes compared to healthy subjects [9]. No differences in $A_{2 A}$ receptor parameters were detected in relation to age, age at onset, or disease duration strata. Patients with a greater density of $\mathrm{A}_{2 \mathrm{~A}}$ receptors were more likely to experience motor complications [9]. In the present study, earlier onset of dyskinesia correlated with the effectiveness of istradefylline; however, the duration of dyskinesia was unrelated. Accordingly, the daily levodopa dose, levodopa equivalent dose, and the levodopa cumulative dose were not linked to the effectiveness of the adenosine $A_{2 \mathrm{~A}}$ antagonist istradefylline in this study.

Previous studies did not identify increased responsiveness to istradefylline in female patients with $\mathrm{PD}$. The adenosine $\mathrm{A}_{2 \mathrm{~A}}$ receptor antagonist ATL $444 \mathrm{dem}$ onstrated reduced sensitivity in male compared to female rats [10]. However, the mechanisms underlying sex-related differences in adenosine $\mathrm{A}_{2 \mathrm{~A}}$ receptor sensitivity were not identified, although ovarian hormones are reported to increase the sensitivity of the D2/A2A receptor system in females [11]. The efficacy on the reduction of daily Off-time shown in four of the five randomized controlled, double blinded, multicenter trials (Table 3) [12] [13] [14] [15]. A Japanese phase III trial that included more female subjects than the western trial demonstrated

Table 3. Off-time change from baseline of the randomized multicenter trials of Istradefylline.

\begin{tabular}{|c|c|c|c|c|c|c|c|c|c|c|c|c|c|}
\hline & & \multirow[b]{2}{*}{$\mathrm{n}$} & \multicolumn{3}{|c|}{ Placebo } & \multicolumn{4}{|c|}{$20 \mathrm{mg}$} & \multicolumn{4}{|c|}{$40 \mathrm{mg}$} \\
\hline & & & $\begin{array}{c}\text { Mean age } \\
\text { (SD) }\end{array}$ & Male \% & $\begin{array}{l}\text { Change } \\
\text { of OFF } \\
\text { time } \\
\text { (hours) }\end{array}$ & $\begin{array}{c}\text { Mean age } \\
(\mathrm{SD})\end{array}$ & Male \% & $\begin{array}{l}\text { Change of } \\
\text { OFF time } \\
\text { (hours) }\end{array}$ & $p$-value & $\begin{array}{c}\text { Mean age } \\
(\mathrm{SD})\end{array}$ & Male \% & $\begin{array}{l}\text { Change } \\
\text { of OFF } \\
\text { time } \\
\text { (hours) }\end{array}$ & $p$-value \\
\hline $\begin{array}{c}\text { Hauser RA. } \\
\text { et al. }[13]\end{array}$ & 2008 & 231 & $64(10.2)$ & 67.0 & -0.9 & $63(9.5)$ & 66.1 & -1.6 & 0.03 & - & - & - & - \\
\hline $\begin{array}{c}\text { Pourcher E. } \\
\text { et al. [14] }\end{array}$ & 2012 & 584 & $63(8.3)$ & 64.2 & -1.3 & $64(9.8)$ & 69.1 & -1.1 & - & $63(9.3)$ & 65.8 & -1.5 & 0.529 (overall) \\
\hline $\begin{array}{c}\text { Mizuno Y. } \\
\text { et al. [2] }\end{array}$ & 2013 & 373 & $65.8(8.6)$ & 47.2 & -0.23 & $66.1(8.6)$ & 33.3 & -0.99 & 0.003 & $65.7(9.0)$ & 52.0 & -0.96 & 0.003 \\
\hline
\end{tabular}


a reduction in daily Off-time in the istradefylline group [2]. In a western Phase III trial, KW-6002-US-018, a reduction in Off-time was -1.3 hours in the placebo group, and it was -1.1 hours in the $20 \mathrm{mg}$ of istradefylline group [14], and their baseline characteristics were similar to the present study, excluding those regarding race and sex. Although, other two Western studies including the same ratio of sex, showed the efficacy on the reduction of Off-time [12] [13] (Table 3).

This study featured several limitations. Primarily, the present study featured a small population; therefore, a larger study will be required to validate these results. Second, this was an open study, and it was not possible to rule out the placebo effect. In addition, the previous Japanese phase III trial revealed a $0.28 \mathrm{~h}$ increase in On-time, and a $0.23 \mathrm{~h}$ reduction in Off-time in the placebo group, therefore, additional effects need to be explored to elucidate this [2]. The placebo effects in female subjects are described in one report, which stated that female subjects with both lower dispositional anxiety and cortisol levels showed the largest vasopressin-induced modulation of placebo effects [16]. However, Shetty et al. analyzed the placebo effects in PD from 22 reports and a DATATOP study and found no correlation with age and gender [17]. In addition, Goetz et al. reported that gender, age, disease duration, and baseline disability score did not influence the likelihood of improvement in association with placebo treatment [18].

\section{Conclusion}

In summary, our results cannot deny placebo effects; however, this study suggests that younger or female patients who are not excessively sleepy during the daytime are better candidates for istradefylline therapy.

\section{References}

[1] Mori, A. and Shindou, T. (2003) Modulation of GABAergic Transmission in the Striatopallidal System by Adenosine $\mathrm{A}_{2 \mathrm{~A}}$ Receptors: A Potential Mechanism for the Antiparkinsonian Effects of $\mathrm{A}_{2 \mathrm{~A}}$ Antagonists. Neurology, 61, S44-S48. https://doi.org/10.1212/01.WNL.0000095211.71092.A0

[2] Mizuno, Y. and Kondo, T., Japanese Istradefylline Study Group (2013) Adenosine $\mathrm{A}_{2 \mathrm{~A}}$ Receptor Antagonist Istradefylline Reduces Daily OFF Time in Parkinson's Disease. Movement Disorders, 28, 1138-1141. https://doi.org/10.1002/mds.25418

[3] Hughes, A.J., Daniel, S.E., Kilford, L. and Lees, A.J. (1992) Accuracy of Clinical Diagnosis of Idiopathic Parkinson's Disease: A Clinico-Pathological Study of 100 Cases. Journal of Neurology, Neurosurgery \& Psychiatry, 55, 181-184. https://doi.org/10.1136/jnnp.55.3.181

[4] Fahn, S. and Elton, R., Members of the UPDRS Development Committee (1987) In: Fahn, S., Marsden, C.D., Calne, D.B. and Goldstein, M., Eds., Recent Developments in Parkinson's Disease, Vol. 2, Macmillan Healthcare Information, Florham Park, 153-163.

[5] Johns, M.W. (1991) A New Method for Measuring Daytime Sleepiness: The Epworth Sleepiness Scale. Sleep, 14, 540-545.

[6] Tomlinson, C.L., Stowe, R., Patel, S., Rick, C., Gray, R. and Clarke, C.E. (2010) Sys- 
tematic Review of Levodopa Dose Equivalency Reporting in Parkinson's Disease. Movement Disorders, 25, 2649-2653. https://doi.org/10.1002/mds.23429

[7] Mishina, M., Ishiwata, K., Naganawa, M., Kimura, Y., Kitamura, S., Suzuki, M., Mishina, M., Ishiwata, K., Naganawa, M., Kimura, Y. and Kitamura, S. (2011) Adenosine $\mathrm{A}_{2 \mathrm{~A}}$ Receptors Measured with $\left[{ }^{11} \mathrm{C}\right] \mathrm{TMSX}$ PET in the Striata of Parkinson's Disease Patients. PLOS ONE, 6, e17338. https://doi.org/10.1371/journal.pone.0017338

[8] Huang, Z.L., Qu, W.M., Eguchi, N., Chen, J.F., Schwarzschild, M.A., Fredholm, B.B., Urade, Y. and Hayaishi, O. (2005) Adenosine $A_{2 A}$, but Not $A_{1}$, Receptors Mediate the Arousal Effect of Caffeine. Nature Neuroscience, 8, 858-859.

https://doi.org/10.1038/nn1491

[9] Casetta, I., Vincenzi, F., Bencivelli, D., Corciulo, C., Gentile, M., Granieri, E., Borea, P.A. and Varani, K. (2014) $\mathrm{A}_{2 \mathrm{~A}}$ Adenosine Receptors and Parkinson's Disease Severity. Acta Neurologica Scandinavica, 129, 276-281.

https://doi.org/10.1111/ane.12181

[10] Doylea, S.E., Breslina, F.J., Riegerb, J.M., Beaugleholeb, A. and Lyncha, W.J. (2012) Time and Sex-Dependent Effects of an Adenosine $\mathrm{A}_{2 \mathrm{~A}} / \mathrm{A}_{1}$ Receptor Antagonist on Motivation to Self-Administer Cocaine in Rats. Pharmacology Biochemistry and Behavior, 102, 257-263.

[11] Bazzett, T.J. and Becker, J.B. (1994) Sex Differences in the Rapid and Acute Effects of Estrogen on Striatal D2 Dopamine Receptor Binding. Brain Research, 637, 163172.

[12] LeWitt, P.A., Guttman, M., Tetrud, J.W., Tuite, P.J., Mori, A., Chaikin, P. and Sussman, N.M., 6002-US-005 Study Group (2008) Adenosine $A_{2 A}$ Receptor Antagonist Istradefylline (KW-6002) Reduces “off” Time in Parkinson's Disease: A Double-Blind, Randomized, Multicenter Clinical Trial (6002-US-005). Annals of Neurology, 63, 295-302. https://doi.org/10.1002/ana.21315

[13] Hauser, R.A., Shulman, L.M., Trugman, J.M., Roberts, J.W., Mori, A., Ballerini, R. and Sussman, N.M., Istradefylline 6002-US-013 Study Group (2008) Study of Istradefylline in Patients with Parkinson's Disease on Levodopa with Motor Fluctuations. Movement Disorders, 23, 2177-2185. https://doi.org/10.1002/mds.22095

[14] Pourcher, E., Fernandez, H.H., Stacy, M., Mori, A., Ballerini, R. and Chaikin, P. (2012) Istradefylline for Parkinson's Disease Patients Experiencing Motor Fluctuations: Results of the KW-6002-US-018 Study. Parkinsonism and Related Disorders, 18, 178-184. https://doi.org/10.1016/j.parkreldis.2011.09.023

[15] Mizuno, Y., Hasegawa, K., Kondo, T., Kuno, S. and Yamamoto, M., The Japanese Istradefylline Study Group (2010) Clinical Efficacy of Istradefylline (KW-6002) in Parkinson's Disease: A Randomized, Controlled Study. Movement Disorders, 25, 1437-1443. https://doi.org/10.1002/mds.23107

[16] Colloca, L., Pine, D.S., Ernst, M., Miller, F.G. and Grillon, C. (2016) Vasopressin Boosts Placebo Analgesic Effects in Women: A Randomized Trial. Biological Psychiatry, 79, 794-802. https://doi.org/10.1016/j.biopsych.2015.07.019

[17] Shetty, N., Friedman, J.H., Kieburtz, K., Marshall, F.J. and Oakes, D. (1999) The Placebo Response in Parkinson's Disease. Parkinson Study Group. Clinical Neuropharmacology, 22, 207-212.

[18] Goetz, C.G., Leurgans, S., Raman, R. and Stebbins, G.T. (2000) Objective Changes in Motor Function during Placebo Treatment in PD. Neurology, 54, 710-714. https://doi.org/10.1212/WNL.54.3.710 
Submit or recommend next manuscript to SCIRP and we will provide best service for you:

Accepting pre-submission inquiries through Email, Facebook, LinkedIn, Twitter, etc. A wide selection of journals (inclusive of 9 subjects, more than 200 journals)

Providing 24-hour high-quality service

User-friendly online submission system

Fair and swift peer-review system

Efficient typesetting and proofreading procedure

Display of the result of downloads and visits, as well as the number of cited articles Maximum dissemination of your research work

Submit your manuscript at: http://papersubmission.scirp.org/

Or contact apd@scirp.org 